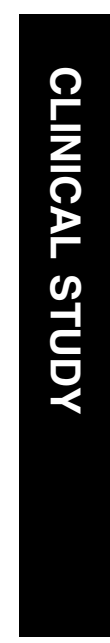

\section{Comparing approaches to screening for angle closure in older Chinese adults}

${ }^{1}$ Johns Hopkins Bloomberg School of Public Health, Baltimore, MD, USA

${ }^{2}$ Wilmer Eye Institute, Johns Hopkins School of Medicine, Baltimore, MD, USA

\section{${ }^{3}$ Zhongshan Ophthalmic Center, Sun Yat-Sen University, Guangzhou, China}

${ }^{4}$ NIHR Biomedical Research Centre for Ophthalmology, UCL Institute of Ophthalmology and Moorfields Eye Hospital, London, UK

\section{${ }^{5}$ Department of Ophthalmology, University of Yamanashi, Yamanashi, Japan}

Correspondence: $\mathrm{M} \mathrm{He}$, Department of Preventive Ophthalmology, Zhongshan Ophthalmic Center, Sun Yat-Sen University, Guangzhou 510060, China Tel: + 862087331109 ; Fax: + 862087331903 . E-mail: mingguanghe@ gmail.com

Received: 21 February 2011 Accepted in revised form: 9 August 2011 Published online: 14 October 2011

\section{Abstract}

Aims Primary angle-closure glaucoma is expected to account for nearly $50 \%$ of bilateral glaucoma blindness by 2020 . This study was conducted to assess the performance of the scanning peripheral anterior chamber depth analyzer (SPAC) and limbal anterior chamber depth (LACD) as screening methods for angle closure.

Methods This study assessed two clinical populations to compare SPAC, LACD, and gonioscopy: the Zhongshan Angle-closure Prevention Trial, from which 370 patients were eligible as closed-angle participants and the Liwan Eye Study, from which 72 patients were selected as open-angle controls. Eligible participants were assessed by SPAC, LACD, and gonioscopy.

Results Angle status was defined by gonioscopy. Area under the receiver operating characteristic curve (AUROC) for SPAC was $0.92(0.89-0.95)$ whereas AUROC for LACD was 0.94 (0.92-0.97). Using conventional cutoff points, sensitivity/specificity was 93.0\%/70.8\% for SPAC and $94.1 \% / 87.5 \%$ for LACD. Sequential testing using both SPAC and LACD increased the specificity to $94.4 \%$ and decreased the sensitivity to $87.0 \%$.

Conclusion SPAC has significantly lower specificity than LACD measurement using conventional cutoffs but interpretation of the findings can be performed by modestly trained personnel.

Eye (2012) 26, 96-100; doi:10.1038/eye.2011.244; published online 14 October 2011

Keywords: glaucoma; angle closure; diagnostic techniques; ophthalmological; gonioscopy
J Andrews' ${ }^{1}$, DS Chang ${ }^{1,2,3}$, Y Jiang ${ }^{3}, \mathrm{M} \mathrm{He}^{3}$, PJ Foster ${ }^{4}$, B Munoz $^{1,2}$, K Kashiwagi $^{5}$ and DS Friedman ${ }^{1,2,3}$

\section{Introduction}

Primary angle-closure glaucoma (PACG) is preventable, yet is a leading cause of blindness globally. ${ }^{1-3}$ In all, $87 \%$ of those with angleclosure glaucoma are located in Asia where two million Chinese residents are blind in at least one eye due to PACG. ${ }^{2}$ PACG is expected to account for nearly half of bilateral glaucoma blindness globally by $2020 .{ }^{4}$

Devices are now available to rapidly screen for angle closure. Understanding the efficacy of these devices will be an important step in developing cost-effective and safe screening strategies to prevent PACG.

The scanning peripheral anterior chamber depth analyzer (SPAC) (Takagi, Nagano, Japan) consists of a non-contact, autofocusing laserbeam outfitted in a portable box that obtains 21 consecutive slit lamp images of the anterior chamber from optical axis to limbus. ${ }^{5}$ It is simple to operate and highly reproducible. ${ }^{6}$

Previous studies have examined the diagnostic capabilities of SPAC. Kashiwagi and Tsukahara $^{7}$ reported $87 \%$ sensitivity and $80 \%$ specificity for diagnosing angle closure when using limbal anterior chamber depth (LACD) as the gold standard. The same researchers showed that SPAC correlates well with traditional anterior chamber angle (ACA) measurement methods, such as the Shaffer grading system, and ultrasound biomicroscopy. ${ }^{8}$ However two studies have noted that SPAC has relatively low specificity when using gonioscopy as the gold standard..$^{8,10}$

Despite this potential criticism, SPAC may still have a role to play due to its high sensitivity. The present study assessed the effectiveness of the SPAC in diagnosing angle closure using gonioscopy as the reference standard in a community-based screening. The large number of cases included in this study is 
novel, and the use of gonioscopy allows comparison across previous and future testing. Evaluating SPAC when used in conjunction with LACD offers new data for researchers and clinicians.

\section{Materials and methods}

Included persons who were primary angle-closure suspects (PACS) were identified as part of the Zhongshan Angle Closure Prevention (ZAP) trial. A detailed study protocol has been published previously. ${ }^{11}$ To be included as PACS, participants over 50 years of age had to have two or more quadrants closed on gonioscopy without peripheral anterior synechiae, elevated intraocular pressure, or glaucomatous optic neuropathy. Participants were excluded if they had prior intraocular surgery, excessively high risk of acute angle-closure attack, or cataract-preventing anterior-chamber imaging. Of 10083 individuals screened for participation in the ZAP trial, $735(7.3 \%)$ were categorized as being bilateral PACS. Only the right eye was included in the analysis (for both cases and controls). Complete testing including SPAC was performed on the first half of these, or 370 individuals, thus 370 were included in this analysis. We believe the potential selection bias incurred by including only half of PACS candidates was minimal because the first half of participants were likely similar to the second half.

Open-angle participants, or those who did not meet the broad PACS criteria outlined above, were selected from the Liwan Eye Study, a population-based prevalence study of eye disease. ${ }^{12}$ For this study, a consecutive series of individuals over 50 years of age who had no evidence of angle closure on gonioscopy was selected under a protocol similar to that used in the ZAP trial. Of 90 participants enrolled from the Liwan Eye Study, 72 open-angle participants for whom complete data were recorded were selected to serve as controls.

Both populations are from Guangzhou and data for both were collected between 2008 and 2010. IRB approval for both the Liwan and ZAP trials was obtained through the Zhongshan Ophthalmic Center, Moorfields Eye Hospital, and the Johns Hopkins University School of Medicine. The ZAP trial is also registered on http:/ / www.controlled-trials.com as ISRCTN45213099.

All study participants underwent a comprehensive ophthalmic examination. A research nurse performed all SPAC testing in ambient light. Subsequently a trained and certified ophthalmologist completed LACD testing. A separate trained ophthalmologist performed the gonioscopy after completion of SPAC and LACD testing. Gonioscopy was performed in the lowest possible ambient light setting.
SPAC measurements ranged from 1 to 12 , with 1 representing the shallowest anterior chamber. The LACD of each eye was evaluated by a modified van Herick grading system using a slit lamp (BQ-900, Haag-Streit, Koniz, Switzerland). ${ }^{13}$ LACD was graded as a percentage fraction of adjacent corneal thickness at the temporal limbus in the following categories: $>100,75,40,25,15$, 5 , and $0 \% .^{14}$

Gonioscopy was performed by a glaucoma-trained ophthalmologist who was found to have high reliability when compared with other ophthalmologists and who had carried out angle-closure research for over a decade $(\kappa>0.8)$. Static gonioscopy was performed, angle width of four quadrants was evaluated using the Shaffer grading system, ${ }^{15}$ and the iris insertion was assessed according to the grading scheme of Spaeth. ${ }^{16}$

Gonioscopy was defined as the reference standard to assess the screening performance of SPAC and LACD. Receiver operator characteristic (ROC) curves for classifying angle closure were generated for SPAC and LACD. Area under the ROC curve (AUROC) was used as an index of global test performance.

In sequential testing, an eye was only classified as angle closure if both SPAC and LACD were positive. Conventional cut points were chosen for each instrument, such that a result of $>6$ on SPAC or $>25 \%$ on LACD was categorized as open angle. ${ }^{9,17}$ Parallel testing was assessed by assuming that a subject was positive if either SPAC or LACD detected angle closure, therefore the point closest to the upper left corner of the ROC curve served as the cutoff point as it generated the best combination of sensitivity and specificity.

Sensitivities and specificities were calculated for all paired testing and were compared using McNemar's test. Positive and negative predictive values were calculated for each single test and the pair based on the $10.2 \%$ prevalence of angle closure within the population of choice in China. ${ }^{12}$ Statistical analysis was completed using STATA 10.1 (StataCorp. LP, College Station, TX, USA). We certify that all applicable institutional and governmental regulations concerning the ethical use of human volunteers were followed during this research.

\section{Results}

A total of 442 participants, including 370 angle-closure cases from the ZAP trial and 72 open-angle controls from the Liwan follow-up study completed all tests. Table 1 demonstrates the predicted baseline differences between ZAP and Liwan study participants, including shallower anterior chamber depth, shorter axial length, and a greater percentage of females among PACS.

Both devices yielded 94\% sensitivity. SPAC had lower specificity $(70.8 \%)$ than LACD $(87.5 \%$, Table 2$)$. 
Table 1 Baseline characteristics and ocular biometrics of study participants in ZAP trial and Liwan study

\begin{tabular}{lcrr}
\hline & ZAP (PACS), $n=370$ & Liwan (control), $n=72$ & P-value \\
\hline Age (year, mean (SD)) & $59.7(5.2)$ & $60.2(3.2)$ & 0.472 \\
Female (no. (\%)) $_{\text {Mean arterial pressure }}$ (mmHg, mean (SD)) & $303(81.89)$ & $42(58.33)$ & $<0.001$ \\
Spherical equivalent $^{\mathrm{b}, \mathrm{c}}$ (Diopter, mean (SD)) & $95.5(12.1)$ & $96.0(15.5)$ & 0.736 \\
Central ACD $^{\mathrm{b}, \mathrm{d}}$ (mm, mean (SD)) & $+1.95(1.34)$ & $+1.13(1.92)$ & $<0.001$ \\
Axial length $^{\mathrm{b}, \mathrm{d}}$ (mm, mean (SD)) & $2.53(0.21)$ & $2.87(0.31)$ & $<0.001$ \\
Lens thickness $^{\mathrm{b}, \mathrm{d}}$ (mm, mean (SD)) & $22.52(0.75)$ & $23.52(1.02)$ & $4.73(0.39)$ \\
Baseline IOP $^{\mathrm{b}}$ (mmHg, mean (SD)) & $4.88(0.34)$ & $14.5(2.1)$ & 0.001 \\
Quadrants of angle closure $^{\mathrm{b}}$ (mean (SD)) & $15.4(2.9)$ & $0.1(0.3)$ & 0.001 \\
SPAC $^{\mathrm{b}, \mathrm{e}}$ (mean (SD)) & $3.4(0.7)$ & $7.86(2.01)$ & 0.011 \\
LACD $^{\mathrm{b}}$ (\%, mean (SD)) & $4.46(1.34)$ & $54(25)$ & $<0.001$ \\
\hline
\end{tabular}

Abbreviations: ACD, anterior chamber depth; LACD, limbal anterior chamber depth; SPAC, scanning peripheral anterior chamber depth analyzer. ${ }^{\mathrm{a}}$ Mean arterial pressure $=$ diastolic blood pressure $+1 / 3$ (systolic blood pressure-diastolic blood pressure).

${ }^{\text {b}}$ Right eye only.

'Spherical equivalent $=$ spherical $+\frac{1}{2}$ cylinder.

${ }^{\mathrm{d}}$ Measured by A scan.

$\mathrm{e}_{n}=370$ in ZAP and $n=72$ in Liwan study completed SPAC.

Table 2 Accuracy of SPAC and LACD against gonioscopy

\begin{tabular}{lccc}
\hline & SPAC $^{\mathrm{a}}(95 \% C I)$ & LACD $^{\mathrm{b}}(95 \%$ CI) & P-value \\
\hline AUROC & $0.92(0.89-0.95)$ & $0.94(0.92-0.97)$ & 0.299 \\
Sensitivity (\%) & $93.0(89.9-95.4)$ & $94.1(91.1-96.2)$ & 0.564 \\
Specificity (\%) & $70.8(58.9-81.0)$ & $87.5(77.6-94.1)$ & 0.011 \\
\hline
\end{tabular}

Abbreviation: $\mathrm{CI}$, confidence interval.

aSPAC cutoff $\leq 6$

${ }^{\mathrm{b}} \mathrm{LACD}$ cutoff $\leq 25 \%$.

Table 3 Sequential and parallel combination of SPAC and LACD against gonioscopy

\begin{tabular}{lcc}
\hline & $\begin{array}{c}\text { Sequential SPAC+ } \\
\text { LACD }\end{array}$ & $\begin{array}{l}\text { Parallel SPAC }+ \\
\text { LACD }^{\mathrm{b}}(95 \% C I)\end{array}$ \\
\hline Sensitivity & $87.0(83.2-90.3)$ & $97.6(95.4-98.9)$ \\
Specificity & $94.4(86.4-98.5)$ & $87.5(77.6-94.1)$ \\
\hline
\end{tabular}

Abbreviation: CI, confidence interval.

aSPAC cutoff $\leq 6$; LACD cutoff $\leq 25 \%$.

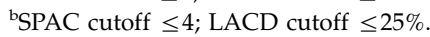

Negative predictive value was greater than $99 \%$ for both devices; positive predictive value was higher for LACD (46\%) than SPAC $(27 \%)$.

Parallel testing resulted in enhanced sensitivity of $97.6 \%$, and specificity that was equivalent to LACD specificity at $87.5 \%$. Sequential testing resulted in increased specificity $(94.4 \%)$ and reduced sensitivity (87.0\%, Table 3). Positive predictive value was $64 \%$ with sequential testing and $47 \%$ with parallel testing; negative predictive value remained $\sim 99 \%$ for each combined testing situation.

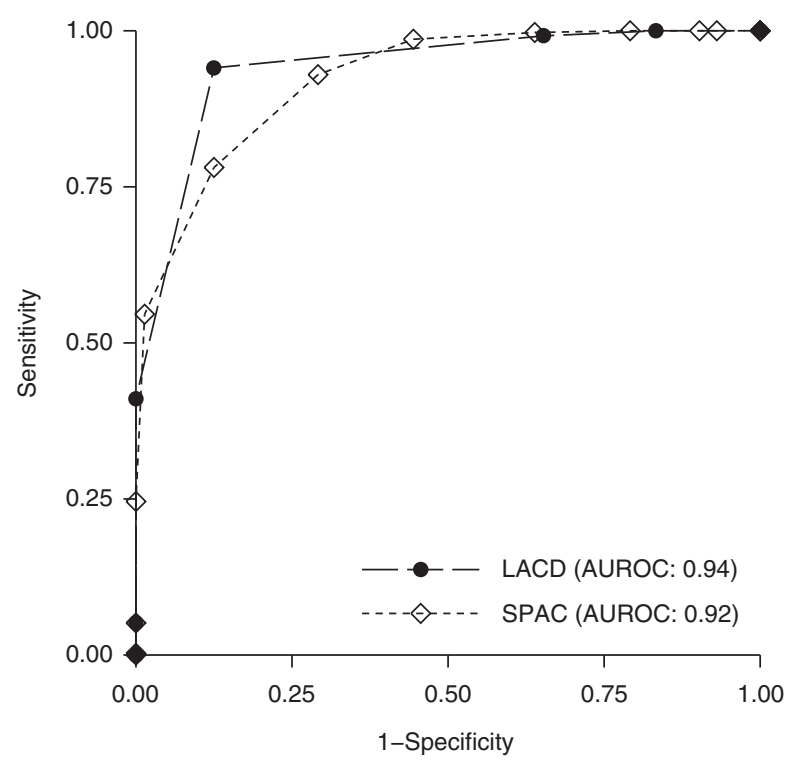

Figure 1 ROC curve for scanning peripheral anterior chamber depth analyzer and limbal anterior chamber depth as compared with the gold standard of gonioscopy.

To look at overall performance of the two approaches, ROC curves for each were analyzed and can be seen in Figure 1 and Table 2. Each had a relatively high area AUROC, at 0.92 for SPAC and 0.94 for LACD. These curves were not statistically significant different $(P=0.30)$. Interestingly, the area under the curve for SPAC was identical to that demonstrated by Kashiwagi et al in another SPAC analysis. ${ }^{7}$

To further demonstrate the diagnostic performance of each test, Figures 2 and 3 illustrate the agreement between each test individually and gonioscopy, with dots representing participants. 


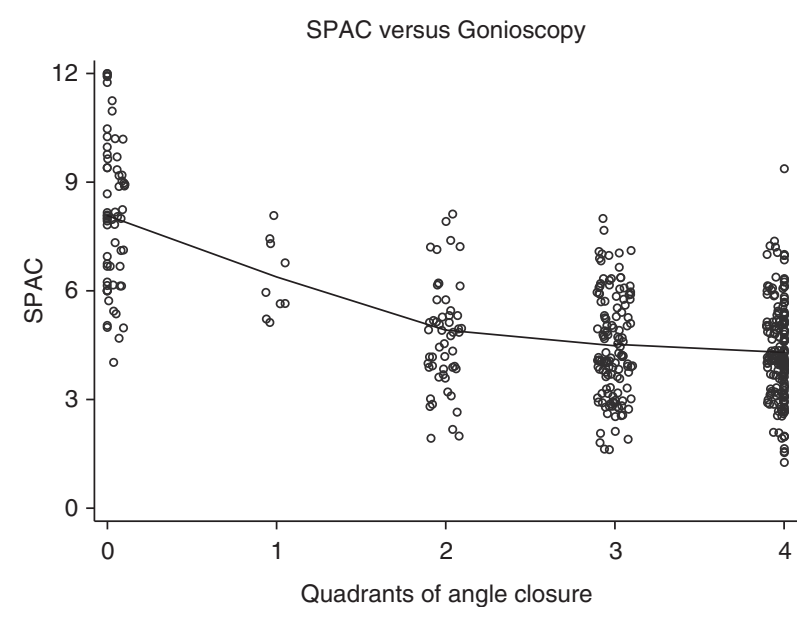

Figure 2 Scatter plot of participants, SPAC vs gonioscopy.

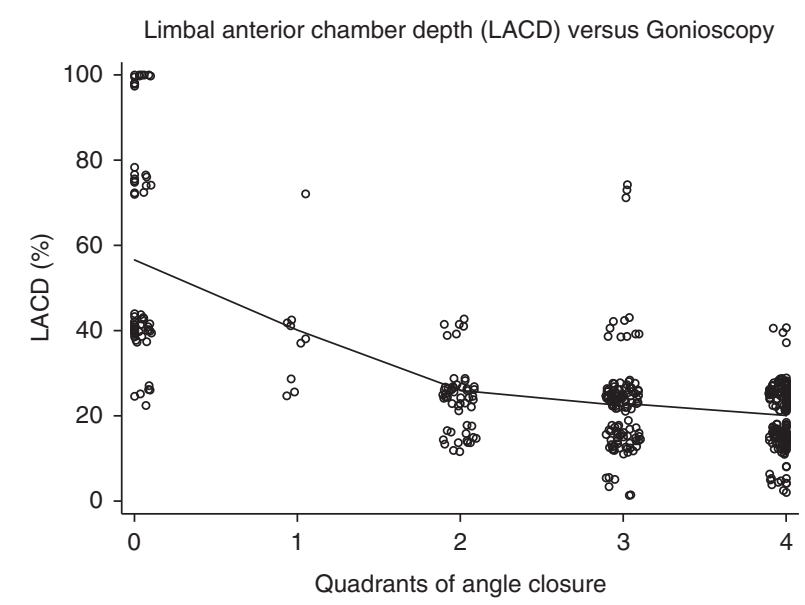

Figure 3 Scatter plot of participants, LACD vs gonioscopy.

\section{Discussion}

SPAC and LACD had similar screening performance when compared with gonioscopy in this large sample of subjects with PACS and open-angle controls. The statistical benefit of adding SPAC for the identification of persons with angle closure was marginal, however, clinically using SPAC and LACD in parallel would identify a greater proportion of PACS.

In a population-based screening setting, however, specificity would be of greater concern. In this case, requiring both tests to be positive would increase specificity to $94 \%$. Depending on the situation, this tradeoff may be worth making to reduce the burden of providing definitive exams. Both tests are easily performed and provide immediate results, making them logistically appealing for screening. Recent research has shown that half of those diagnosed as having glaucoma in the United States never had gonioscopy documented in the chart, making alternatives increasingly important. ${ }^{18}$

A few minor limitations should be considered. PACS and open-angle participants were chosen from different studies and had different baseline characteristics as shown in Table 1. These differences were expected, thus no adjustments were made in later analyses.

Additionally, those with more severe forms of angle closure were not included in this study. ${ }^{12}$ Including more severe forms of angle closure such as primary angle closure and PACG subjects should increase the sensitivity, specificity, and overall utility of the tools being tested, if causing any change. Lastly SPAC was developed in a Japanese cohort but used in a Chinese population in this study. Although SPAC has been studied in Chinese populations, one might presume that performance of the test could improve further if the internal algorithms of SPAC were adjusted for use in Chinese populations.

In summary, SPAC does not offer diagnostic superiority over current methods, perhaps because it uses light in the visible spectrum and this may open the limbal angle as miosis occurs. Measurement of LACD is simple and can be done with standard ophthalmic equipment. SPAC is an expensive, separate piece of equipment that only diagnoses risk status for one disease, whereas traditional slit-lamp technology allows for a complete eye examination and diagnosis of multiple pathologies. No study has assessed whether technicians can be trained to accurately perform LACD, which could alter discussions about human resource availability in considering whether to use SPAC or LACD. Large screening situations may be ideal for combined SPAC and LACD measurement as requiring both to be positive would substantially reduce the false positive referral rate with only a small decrease in the overall sensitivity.

\section{Summary}

\section{What was known before}

- The scanning peripheral angle chamber depth analyzer (SPAC) has shown reasonable sensitivity/specificity $(87 \% / 80 \%)$ in one prior study using limbal anterior chamber depth (LACD) as the gold standard. SPAC has been shown to have reasonable correlation with anterior chamber angle measurement methods, and was reasonably well correlated to gonioscopy measurements in one study.

\section{What this study adds}

- This study utilizes three modalities, LACD, gonioscopy, and SPAC to ascertain the sensitivity of SPAC and LACD both alone and simultaneously, in parallel or in series. These permutations allow the authors to think anew about the practicalities of using SPAC in particular as a diagnostic tool for clinical or research purposes. 


\section{Conflict of interest}

Dr Kashiwagi has a Japanese patent on the SPAC (Japanese patent No. 3878164). Dr Friedman currently has the SPAC instrument on loan from Carl Zeiss Meditec. The remaining authors declare no conflict of interest.

\section{Acknowledgements}

This study was funded by Fight for Sight (London), and the Richard Desmond Charitable Foundation via Fight for Sight, the City of Guangzhou, and private donations from Johns Hopkins affiliates.

\section{References}

1 Quigley HA, Broman AT. The number of people with glaucoma worldwide in 2010 and 2020. Br J Ophthalmol 2006; 90: 262-267.

2 Foster PJ, Johnson GJ. Glaucoma in China: how big is the problem? Br J Ophthalmol 2001; 85: 1277-1282.

3 Resnikoff S, Pascolini D, Etya'ale D, Kocur I, Pararajasegaram R, Pokharel GP et al. Global data on visual impairment in the year 2002. Bull World Health Organ 2004 82: 844-851.

4 See JL. Imaging of the anterior segment in glaucoma. Clin Exp Ophthalmol 2009; 37: 506-513.

5 Lavanya R, Teo L, Friedman DS, Aung HT, Baskaran M, Gao $\mathrm{H}$ et al. Comparison of anterior chamber depth measurements using the IOLMaster, scanning peripheral anterior chamber depth analyser, and anterior segment optical coherence tomography. Br J Ophthalmol 2007; 91: 1023-1026.

6 Kashiwagi K, Kashiwagi F, Toda Y, Osada K, Tsumura T, Tsukahara S. A newly developed peripheral anterior chamber depth analysis system: principle, accuracy, and reproducibility. Br J Ophthalmol 2004; 88: 1030-1035.

7 Kashiwagi K, Tsukahara S. Case finding of angle closure glaucoma in public health examination with scanning peripheral anterior chamber depth analyzer. J Glaucoma 2007; 16: 589-593.
8 Kashiwagi K, Tsumura T, Tsukahara S. Comparison between newly developed scanning peripheral anterior chamber depth analyzer and conventional methods of evaluating anterior chamber configuration. J Glaucoma 2006; 15: $380-387$.

9 Friedman DS, He M. Anterior chamber angle assessment techniques. Surv Ophthalmol 2008; 53: 250-273.

10 Lavanya R, Foster PJ, Sakata LM, Friedman DS, Kashiwagi $\mathrm{K}$, Wong TY et al. Screening for narrow angles in the singapore population: evaluation of new noncontact screening methods. Ophthalmology 2008; 115: 1720-1727, 1727.e1-e2.

11 Jiang Y, Friedman DS, He M, Huang S, Kong X, Foster PJ. Design and methodology of a randomized controlled trial of laser iridotomy for the prevention of angle closure in southern China: the Zhongshan angle Closure Prevention trial. Ophthalmic Epidemiol 2010; 17: 321-332.

12 He M, Foster PJ, Ge J, Huang W, Zheng Y, Friedman DS et al. Prevalence and clinical characteristics of glaucoma in adult Chinese: a population-based study in Liwan District, Guangzhou. Invest Ophthalmol Vis Sci 2006; 47: 2782-2788.

13 van Herick W, Shaffer RN, Schwartz A. Estimation of width of angle of anterior chamber: incidence and significance of the narrow angle. Am J Ophthalmol 1969; 68: 626-629.

14 Foster PJ, Devereux JG, Alsbirk PH, Lee PS, Uranchimeg D, Machin D et al. Detection of gonioscopically occludable angles and primary angle closure glaucoma by estimation of limbal chamber depth in Asians: modified grading scheme. Br J Ophthalmol 2000; 84: 186-192.

15 Shaffer RN. Stereoscopic Manual of Gonioscopy. Mosby: St Louis, MO, 1962.

16 Spaeth GL. The normal development of the human anterior chamber angle: a new system of descriptive grading. Trans Ophthalmol Soc UK 2008; 91: 710-739.

17 Baskaran M, Oen F, Chan YH, Hoh ST, Ho CL, Kashiwagi K et al. Comparison of the scanning peripheral anterior chamber depth analyzer and the modified van Herick grading system in the assessment of angle closure. Ophthalmology 2007; 114: 501-506.

18 Quigley HA, Friedman DS, Hahn SR. Evaluation of practice patterns for the care of open-angle glaucoma compared with claims data: the Glaucoma Adherence and Persistency Study. Ophthalmology 2007; 114: 1599-1606. 